\section{OC-027 DOES THE ORGANISATION OF SERVICES IN A GEOGRAPHICALLY DIVERSE REGION HAVE IMPACT UPON DELIVERY OF END OF LIFE CARE FOR PATIENTS WITH UPPER GI CANCER?}

doi:10.1136/gutjnl-2012-302514a.27

A Jones, ${ }^{*}$ R Coggins, J Godsman. NHS Highland, Inverness, UK

Introduction The Scottish government "Living and Dying Well" action plan aims to ensure that end of life care needs are recognised in all care settings. This study aims to examine end of life care for Upper GI cancer patients, diagnosed within the geographically diverse region that comprises NHS Highland. Following diagnosis, do we get patients home, and do they die at home?

Methods E-Case Cancer Audit, Cancer Waiting Times Submissions, General Register Office Death and Cancer Registry databases were searched using ICD10 codes for upper GI cancer (ICD10 C15-C17 and C22-C25) for years 2005-2010. Place of death for patients diagnosed within NHS Highland was identified and recorded as one of: home, hospital, hospice or "other institution". This data were then compared with Scottish national data for all cancers, compiled and published by Information Services Division, Scotland.

Results 978 patients were diagnosed with upper GI cancer in the study period. Place of death was not known in 298 patients and these were therefore excluded from further analysis. Of the remaining 680 patients 237 (34.9\%) died at home, 295 (43.4\%) died in hospital, 96 (14.1\%) died in hospice and 49 (7.2\%) died in another institution. Of 75522 cancer deaths in Scotland between 2004 and $200824.3 \%$ died at home, $51.9 \%$ died in hospital, $17.6 \%$ died in hospice and $6.2 \%$ died in another institution. Using $\chi^{2}$ testing with Yates' correction, differences between NHS Highland and national data show highly significant differences in both "at home" and "in hospital" deaths $(p<0.0001)$.

Conclusion Despite government initiatives, over half of cancer patients in Scotland still die in hospital and just under a quarter die at home. In our study group fewer patients die in hospital and more-over one third-die at home. Despite the challenges of geography and transport links in the north of Scotland, the ability to deliver complex care and end of life planning for patients with Upper GI cancer is not adversely affected.

Competing interests None declared.

\section{REFERENCE}

1. Information Services Division. Living and Dying Well: A National Action Plan For Palliative And End Of Life Care In Scotland. Scotland: NHS National Services, 2008. http://www isdscotland.org. ISBN 9780755958894.

\section{OC-028 DEVELOPING SUSTAINABLE GI ENDOSCOPY TRAINING IN MALAWI}

doi:10.1136/gutjnl-2012-302514a.28

1J Geraghty, ${ }^{*} \mathrm{~A}$ Kankwatira, ${ }^{3} \mathrm{M}$ Feeney, ${ }^{4} \mathrm{M}$ Hendrickse, ${ }^{5} \mathrm{~L}$ Kalongolera, ${ }^{2} \mathrm{R}$ Malamba, ${ }^{2} \mathrm{~N}$ Mtunthama, ${ }^{6} \mathrm{H}$ Mwandumba, ${ }^{1} \mathrm{P} 0$ 'Toole, ${ }^{1} \mathrm{M}$ Gordon. ${ }^{1}$ Department of Gastroenterology, Royal Liverpool University Hospital, Liverpool, UK; ${ }^{2}$ Clinical Investigation Unit, Queen Elizabeth Central Hospital, Blantyre, Malawi; ${ }^{3}$ Department of Gastroenterology, South Devon Healthcare, Torbay; ${ }^{4}$ Department of Gastroenterology, Blackpool Victoria Hospital, Blackpool, UK; ${ }^{5}$ Department of Surgery, Queen Elizabeth Central Hospital, Blantyre, Malawi; ${ }^{6}$ Department of Medicine, Queen Elizabeth Central Hospital, Blantyre, Malawi

Introduction Sustainable endoscopy services could improve management of upper gastrointestinal malignancy and haemorrhage, both common in Malawi. Since 2008 we have committed to improving endoscopy training through an International Health Link (IHL) partnership with Malawi. We aimed to (1) develop a sustainable training "hub" with locally-trained Trainers in Blantyre
(2) develop locally-relevant training courses, (3) extend training support to regional hospital "spokes".

Methods We partnered in five training visits to Malawi, funded by the Tropical Health Education Trust and the British Society of Gastroenterology. We ran 14 courses (Basic Skills, Skills Enhancement, Training the Gastroscopy Trainers (TGT) and Endoscopy Nurses) involving 52 delegate-training-episodes (29 local doctors, 12 clinical officers (COs), three expatriate doctors, eight nurses). Outcomes were monitored by JAG-format DOPS and course evaluations. Progress over time towards the three aims was assessed.

Results Aim 1) Training models and audit, reporting and assessment tools were introduced in Blantyre. The mean number of delegateepisodes increased from 6.3 during the first four visits, to 20 during the last two visits. During the first four visits the local faculty was four expatriate doctors and one $\mathrm{CO}$, increasing to seven local doctors, five COs, two nurses and one expatriate doctor during the last two visits. In the first four visits, 16/21 delegate-episodes involved only skills learning and 5/21 (24\%) were as mentored or local faculty, while in the last two visits, $25 / 40$ involved only skills learning and 15/40 (38\%) were as mentored or local faculty. In 2011 we ran and evaluated the first TGT within Malawi. Aim 2) We developed a Basic Skills in Gastroscopy course appropriate to local circumstances, which was delivered, evaluated and modified over each visit, and ultimately delivered by two locally-trained Trainers. Aim 3) The delegates' region of origin for the first four visits was 18/ 19 from Blantyre, and for the last two visits was 15/40 Blantyre, 13/ 40 Lilongwe, 7/40 Zomba, 3/40 Mzuzu and 2/40 Zambia. The origin of UK external faculty increased from 1 to 3 sites, and two new IHLs were established with Lilongwe and Zomba.

Conclusion IHL partnerships represent a sustainable means of improving GI endoscopy training. Modified JAG-format courses, assessments and evaluations were useful even in a resource-limited setting. A hub-and-spoke model helped to support other regions. Future training priorities include training in therapy and further development of local trainers. Local reporting tools should allow audit of outcomes across regions.

Competing interests None declared.

\section{OC-029 DYSPEPSIA MANAGEMENT IN MALAWI: A PROSPECTIVE AUDIT}

doi:10.1136/gutjnl-2012-302514a.29

${ }^{1} \mathrm{M}$ G Keane, ${ }^{*}{ }^{2} \mathrm{~A}$ Thumbs, ${ }^{2} \mathrm{~K}$ Hellberg, ${ }^{2} \mathrm{~T}$ Allain, ${ }^{2} \mathrm{~A}$ Kankwatira, ${ }^{2} \mathrm{~W}$ Howson, ${ }^{3} \mathrm{~J}$ Geraghty, ${ }^{2} \mathrm{R}$ Malamba, ${ }^{4} \mathrm{M}$ A Gordon, ${ }^{3} \mathrm{P} 0$ 0'Toole. ${ }^{1}$ Department of Gastroenterology, UCLH, London, UK; ${ }^{2}$ College of Medicine, OECH, Blantyre, Malawi; ${ }^{3}$ Department of Gastroenterology, Royal Liverpool University Hospital, UK; ${ }^{4}$ Department of Gastroenterology, University of Liverpool, Liverpool, UK

Introduction Dyspepsia is a very common symptom world wide. Unless managed effectively it can burden endoscopy services and create high treatment costs. Management algorithms are widely used in developed countries but have not been validated in subSaharan Africa. In Malawi, Helicobacter pylori (HP) infection rates are high, endoscopy facilities are scarce and proton pump inhibitors (PPIs) expensive. In addition the region has extremely high rates of oesophageal cancer and HIV. The incidence of gastric cancer is not known. These conditions make dyspepsia management particularly challenging. Queen Elizabeth Central Hospital (OECH) in Blantyre is Malawi's tertiary hospital, and provides endoscopy services for a large part of the Southern Region.

Methods A prospective audit of all patients presenting routinely to the outpatient department or endoscopy unit at $\mathrm{OECH}$ with dyspeptic symptoms over a 4-week period between August and September 2010. Patients were interviewed and health records and prescriptions reviewed. 
Results 105 patients with dyspepsia were identified; 55 already listed for endoscopy. 143 prescriptions for HP eradication were reviewed; $95 \%$ of these did not conform to a recognised regimen. Most errors were in dosing or duration but $38 \%$ used a $\mathrm{H}_{2}$ receptor antagonist instead of a PPI and 31\% included only one antibiotic. The 55 patients undergoing gastroscopy had all received prior HP eradication therapy. Mean symptom duration was 33.8 months. The clinical diagnosis matched endoscopic findings in only $18 \% .9 \%$ were found to have peptic ulcer disease and "gastritis" was recorded for $35 \%$. There was one gastric cancer and 10 oesophageal cancers. Seven of these 11 patients had dysphagia and malignancy had been suspected; 4/11 malignancies were not suspected. Only $5.5 \%$ of endoscopies were normal.

Conclusion Empirical management of dyspepsia in Malawi is poor. $\mathrm{HP}$ eradication therapy is given frequently but almost always incorrectly. This is likely to promote antibiotic resistance and make subsequent HP eradication more difficult. Referral criteria for endoscopy are not clear, yet the yield of serious pathology is surprisingly high. The low rate of normal endoscopic findings contrasts with UK practice but may be explained by over-diagnosis of "gastritis". In light of this audit, guidelines on dyspepsia management were developed and implemented. They emphasise correct medical management in young dyspeptic patients without alarm symptoms and urgent referral for gastroscopy if malignancy is suspected at any age. Urease testing has been piloted. The impact of these changes will be assessed later this year.

Competing interests None declared.

\section{OC-030 BENEFITS OF INTRODUCTION OF A SYMPTOM TRIGGERED REGIMEN FOR MANAGEMENT OF ALCOHOL WITHDRAWAL IN A LARGE TEACHING HOSPITAL TRUST: REDUCED ADMISSION DURATION AND COST SAVINGS}

doi:10.1136/gutjnl-2012-302514a.30

${ }^{1} \mathrm{~T} J \mathrm{~W}$ Lee, ${ }^{*} \mathrm{M}$ Samuel, ${ }^{1} \mathrm{~L}$ Bewick, ${ }^{3} \mathrm{H}$ Rutherford, ${ }^{1} \mathrm{P}$ Perez, ${ }^{4} \mathrm{E}$ Gilvarry, ${ }^{1} \mathrm{M}$ Cunningham, ${ }^{3} \mathrm{C}$ Dipper, ${ }^{1} \mathrm{~S}$ Masson. ${ }^{1}$ Freeman Hospital, Newcastle Upon Tyne, UK; ${ }^{2}$ Newcastle University, Newcastle Upon Tyne, UK; ${ }^{3}$ Royal Victoria Infirmary, Newcastle Upon Tyne, UK; ${ }^{4}$ Addiction Psychiatry, Freeman Hospital, Newcastle Upon Tyne, UK

Introduction Acute alcohol withdrawal (AAW) is an increasingly frequent cause for hospital admission in the UK. NICE guidance (2010) recommends the use of a symptom triggered regimen (STR) rather than a fixed-dose regimen of benzodiazepines for the management of AAW. The aim of this study is to observe the effects of the introduction of a STR protocol as part of the introduction of a specialist Alcohol Liaison Team (ALT) in a large teaching hospital trust.

Methods Data were collected prospectively on all patients admitted for management of AAW during two 3-month periods; before and after the introduction of a symptom triggered regimen. The Clinical institute for Withdrawal Assessment for alcohol scale (CIWA-Ar) was used to assess AAW objectively. The introduction of the STR was overseen by an ALT consisting of a specialist nurse and a consultant at both hospital sites in the trust.

Results In the initial period, 102 patients were managed using a fixed-dose regime. Median length of stay (LoS) was 4.0 days (IOR 2-6 days). Median total chlordiazepoxide dose was $260 \mathrm{mg}$ (IOR $120-490 \mathrm{mg}$ ). After introduction of STR, 119 patients were included. Median length of stay was 3.0 days (IOR 1.5-5.0 days). Median total chlordiazepoxide dose was $200 \mathrm{mg}$ (IOR 55-450 mg). Age, gender and comorbidities in the before and after groups were comparable. The reduction in length of stay and total chlordiazepoxide dosage following introduction of the STR protocol were statistically significant $(p<0.001)$.
Conclusion Introduction of a STR protocol for management of acute alcohol withdrawal was associated with a reduction in the length of stay and total benzodiazepine dose required. A specialist ALT facilitated the introduction of the STR at two hospital sites in a large teaching hospital trust. The reduction in length of stay alone could contribute to potential cost savings to the trust of around $£ 280000$ per annum. This justifies the presence of an alcohol liaison team in the trust and supports the recommendations of the NICE guidelines.

Competing interests None declared.

\section{OC-031 DEVELOPMENT OF A COLLABORATIVE INPATIENT ALCOHOL STRATEGY}

doi:10.1136/gutjnl-2012-302514a.31

${ }^{1} \mathrm{M}$ York-Smith, ${ }^{2} \mathrm{R}$ Thompson, ${ }^{1} \mathrm{G}$ Burrell, ${ }^{3} \mathrm{~S}$ Green, ${ }^{3} \mathrm{~K}$ Phekoo, ${ }^{1} \mathrm{~J}$ Fehler, ${ }^{4} \mathrm{~V}$ P B Grover. ${ }^{*}$ Hillingdon Drugs and Alcohol Service, Central and North West London NHS Foundation Trust, UK; ${ }^{2}$ Acute Medical Unit, The Hillingdon Hospitals NHS Foundation Trust, Middlesex, UK; ${ }^{3}$ NIHR CLAHRC for North West London, Chelsea and Westminster Hospital, London, UK; ${ }^{4}$ Department of Gastroenterology, The Hillingdon Hospitals NHS Foundation Trust, Middlesex, UK

Introduction Alcohol-related medical admissions rise by $10 \%$ every year at The Hillingdon Hospitals NHS Foundation Trust, frequently readmitting patients to the medical wards. We sought to work collaboratively, crossing traditional boundaries between different care providers, to improve the quality of care provided to patients admitted with alcohol-related problems.

Methods Acute and community care providers worked with the Collaboration for Leadership in Applied Health Research and Care (CLAHRC) for Northwest London to identify and overcome barriers to care and communication for patients with alcohol-related admissions to the medical wards at a District General Hospital. We employed a Psychologist with appropriate addiction and alcohol expertise as an Alcohol Specialist Practitioner (ASP). The role of the ASP was to: (1) educate, empower and support staff to better identify patients with alcohol problems deliver; (2) perform a comprehensive psychological assessment; (3) deliver psychological interventions; (4) encourage patient engagement with community alcohol services when assessed as appropriate; (5) measure outcomes as a result of service implementation. 103 medical inpatients (76 male; 27 female) were referred to the new inpatient service between 1 August 2011 and 31 December 2011. We compared the rate of engagement with alcohol services of the cohort referred to the ASP, to a cohort of 41 patients admitted to the Gastroenterology ward in 2010 with an alcohol-related admission, formally referred to alcohol services on discharge, by the Ward Manager. We studied the 30-day readmission rates between patients who did and did not engage with psychological interventions/community services. The Student's t-test was used for statistical analysis between groups.

Results 7 of the 103 patients referred to the service subsequently died as an inpatient, a further seven were abstinent at the time of referral. Analysis was performed on data from the remaining 89 patients. $69.5 \%$ of patients referred to the inpatient service engaged with the ASP and/or community alcohol services. This compares to an engagement rate of $15 \%$ with community alcohol services before the appointment of the ASP, ( $p$ 63\% of those that declined the assessment/intervention of the ASP were readmitted within 30 days, significantly more than intervention group ( $p$

Conclusion Employment of a Psychologist as an ASP on the medical wards significantly improved the subsequent engagement of patients with community alcohol services. Patients engaged with community alcohol services had a significantly lower rate of alcoholrelated readmissions, compared to those who did not engage.

Competing interests None declared. 